\title{
Evidence From Web-Based Dietary Search Patterns to the Role of B12 Deficiency in Non-Specific Chronic Pain: A Large-Scale Observational Study
}

Eitan Giat ${ }^{1}, \mathrm{MD}, \mathrm{PhD}$; Elad Yom-Tov ${ }^{2}, \mathrm{PhD}$

${ }^{1}$ Rheumatology Unit, The Autoimmune Center, Sheba Medical Center, Ramat Gan, Israel

${ }^{2}$ Microsoft Research, Herzeliya, Israel

Corresponding Author:

Elad Yom-Tov, PhD

Microsoft Research

13 Shenkar St

Herzeliya, 46875

Israel

Phone: 972747111359

Email: eladyt@yahoo.com

\section{Abstract}

Background: Profound vitamin B12 deficiency is a known cause of disease, but the role of low or intermediate levels of B12 in the development of neuropathy and other neuropsychiatric symptoms, as well as the relationship between eating meat and B12 levels, is unclear.

Objective: The objective of our study was to investigate the role of low or intermediate levels of B12 in the development of neuropathy and other neuropsychiatric symptoms.

Methods: We used food-related Internet search patterns from a sample of 8.5 million people based in the US as a proxy for B12 intake and correlated these searches with Internet searches related to possible effects of B12 deficiency.

Results: Food-related search patterns were highly correlated with known consumption and food-related searches $(\rho=.69)$. Awareness of B12 deficiency was associated with a higher consumption of B12-rich foods and with queries for B12 supplements. Searches for terms related to neurological disorders were correlated with searches for B12-poor foods, in contrast with control terms. Popular medicines, those having fewer indications, and those which are predominantly used to treat pain, were more strongly correlated with the ability to predict neuropathic pain queries using the B12 contents of food.

Conclusions: Our findings show that Internet search patterns are a useful way of investigating health questions in large populations, and suggest that low B12 intake may be associated with a broader spectrum of neurological disorders than previously thought.

(J Med Internet Res 2018;20(1):e4) doi: 10.2196/jmir.8667

\section{KEYWORDS}

B12 deficiency; diet; Internet searches; neuropsychiatric symptoms; neuropathy

\section{Introduction}

Vitamin B12 has long been known to be a cause of megaloblastic anemia and neurological disorders [1]. Very low levels of B12 cause subacute combined degeneration of the dorsal and lateral spinal columns, manifested by paresthesia, ataxia, impaired sense of vibration and proprioception, progressive weakness, spasticity, and paraplegia. These symptoms may be irreversible, depending on duration. Other neurological abnormalities may be caused by B12 deficiency, such as peripheral neuropathy, memory loss, irritability, and dementia [2,3], but the association of these symptoms with serum B12 levels and B12 intake is unclear. Many cases of B12 deficiency come to medical attention because of these symptoms, but seemingly asymptomatic B 12 deficiency is very common, with prevalences up to $10 \%$ to $25 \%$ [4]. Furthermore, neurological symptoms due to B12 deficiency may appear in patients within a normal range of serum B12 levels [5].

There is no clear cut-off for serum B12 levels [6] — levels higher than $300 \mathrm{pg} / \mathrm{ml}$ are considered normal, whereas between 200 
and $300 \mathrm{pg} / \mathrm{ml}$ are considered borderline, and less than 200 $\mathrm{pg} / \mathrm{ml}$ are considered low. In addition, measurements of serum B12 levels are not always reliable [6]. Several methods are used by different labs, resulting in different normal ranges. Furthermore, results are highly variable, with absolute intra-individual variation less than $100 \mathrm{pg} / \mathrm{ml}$ on repeat testing in one fifth of the patients. Serum B12 concentration may be normal in $5 \%$ of patients with documented B12 deficiency [7].

Intermediate or low levels of B12 may contribute to the development of symptoms that are not typically associated with B12 deficiency. For instance, B12 dietary intake inversely correlated with sleep duration in healthy Japanese patients [8]. B12 levels also inversely correlated with cardiovascular autonomic neuropathy in diabetics (mean level of $289 \mathrm{pg} / \mathrm{ml}$ ), suggesting that intermediate levels may also be clinically significant. Some have suggested low B12 levels may be a risk factor for depression, but evidence is conflicting $[9,10]$.

The effect of diet on B12 levels is not fully understood and depends on food source and bioavailability. Usual western diets contain between 5 to $7 \mathrm{mcg}$ of daily B12 intake, which is similar to the daily recommended intake, while body stores of B12 are 2000 to $5000 \mathrm{mcg}$ [6]. While foods derived from animals are the major dietary sources of B12, supplements containing B12 are commonly used in the United States and many foods are fortified with B12 (eg, non-dairy milks, meat substitutes, and breakfast cereals) [4]. B12 is naturally protein-bound and its absorption depends on gastric acidity and intrinsic factors, which may be influenced by drugs and comorbidities; reduced absorption of unbound B12 found in vitamin supplements is common among healthy patients. Evidence on the effect of B12 intake from meat is confounding. A study assessing 2999 participants from the Framingham Offspring study found meat intake did not affect B12 levels and suggested a protective effect from supplements and B12 fortified foods [4]. In Norway, where cereals are not fortified with B12, dietary intake of B12 from milk and fish was associated with higher plasma vitamin B12 concentrations, whereas B12 from meat or eggs intake was not [11]. In contrary, analysis from the Dutch B-PROOF study [12] suggested that the bioavailability of B12 intake from meat is comparable to B12 intake from milk. To our knowledge, B12 intakes from different types of meat, such as beef, pork or chicken have not been compared.

Internet search activity has been shown to reflect behavior in the physical world [13] since people use such media for information regarding many everyday behaviors, reflecting their diet, habits, and illnesses [14]. In this study, we used these data to assess food consumption and physical symptoms experienced by people using search engines. With respect to food consumption, past work has found a high correlation between recipe searches and actual consumption [15]. The caloric values of recipes saved (bookmarked) on a website [16] and on Twitter [17] were found to be correlated with obesity at US state and county levels. The link between medical symptoms experienced by people and their searches has been used to understand a range of medical phenomena, including the adverse reactions of medical drugs [18], early detection of cancer [19], and precursors to disease [20].
Many symptoms typically associated with B12 deficiency can occur among patients with intermediate to normal levels of B12. We therefore sought to assess the impact of diet on these symptoms. Here, we analyzed Internet searches related to food to quantify dietary B12 intake from different sources of food and their association with B12-related symptoms. We also sought to find a connection between B12 intake and other symptoms that are not typically associated with B12 deficiency, such as chronic pain or fibromyalgia.

\section{Methods}

A list of 212 target terms related to different types of chronic pain were identified from the following categories: (1) antidepressants, (2) neuropathic drugs, (3) other pharmaceuticals (antihistamines and anti-acids), (4) descriptions of pain, (5) disorders related to excess acid, (6) over the counter antacids, (7) psychotherapy, and (8) medical cannabis. The terms are listed in Multimedia Appendix 1. Generic and commercial drug names of these disorders were taken from the Uptodate, Micromedex and drugs.com websites. We also tested 27 medical terms (pharmaceuticals and conditions) which were unlikely to be associated with B12 deficiency and used these as control terms. Control terms were chosen from an online medical dictionary (Medterms). To ensure randomness, we chose the first terms in the alphabetical list, omitting terms which were rarely searched and could bias the results.

We extracted all searches made in English by people in the United States on the Bing search engine during October 2016. For each search, we extracted an anonymized user identifier, the text of the search, and the zip code from which the user made the search. The classes of search categories were (1) recipe searches, searches that contained a reference to a food recipe, as identified by a propriety classifier; (2) term searches, searches that contained 1 or more of the target terms (212 terms above) or the 27 control terms; and (3) B12 searches, searches that contained the term "B12" in them.

To identify ingredients required for each recipe we used the list of recipes from Recipes Wikia [21]. Specifically, we extracted all recipes that contained 1 or more of the following 12 food ingredients (food types), which have a variety of B12 contents: shellfish, mackerel, trout, salmon, tuna, pig, beef, turkey, chicken, egg, milk, and tomatoes. A total of 9449 recipes contained 1 or more of these ingredients.

Additional data required for analysis included the following: (1) B12 contents per serving of the 12 food ingredients, from the US National Institutes of Health Office of Dietary Supplements (Table 1); (2) food spending data from the US Bureau of Labor Statistic's Consumer Expenditure Survey 2015; (3) cost of food items from the US Department of Agriculture's Center for Nutrition Policy and Promotion; (4) food consumption data from the US Department of Agriculture's Economic Research Service; and (5) the number of indications for each medical drug from the Canadian Vigilance Adverse Reactions Online database. All indications reported by at least 10 cases were considered. 
Table 1. B12 content in different types of meat according to the National Institutes of Health Office of Dietary Supplements.

\begin{tabular}{ll}
\hline Meat source & B12 content, mcg/100 grams \\
\hline Shellfish & 98.9 \\
Mackerel & 19 \\
Beef & 6 \\
Trout & 3.8 \\
Salmon & 2.4 \\
Tuna & 1.6 \\
Milk & 0.9 \\
Turkey & 0.8 \\
Egg & 0.6 \\
Pork & 0.4 \\
Chicken & 0.3 \\
\hline
\end{tabular}

The study was deemed exempt by the Microsoft Institutional Review Board.

A demographic analysis of people searching for B12 is provided in Multimedia Appendix 2. The distribution of food searches by US state is given in Multimedia Appendix 3.

\section{Results}

Approximately 8.4 million people searched for a target medical term; the term "B12" or a food recipe was included in the analysis. Of the 212 target medical terms, only 101 were queried by 1000 or more people and were included in the analysis. A total of 3297 recipes were matched to at least 1 query. Manual examination of a random sample of 400 queries found that $87.8 \%(351 / 400)$ of queries were correctly matched to a recipe.

Here, we used searches for food recipes as a proxy for consumption, as suggested by West et al [15]. To further validate that this proxy is accurate, we correlated the fraction of known expenditure on 6 food items (beef, pork, poultry, fish and seafood, eggs, and milk) at each of 4 US regions (Northeast, Midwest, South, and West) reported by the US Bureau of Labor Statistic's Consumer Expenditure Survey 2015 (see Methods), with the number of queries for recipes containing each of these food items weighted by their cost. The Pearson correlation between the 2 was $0.69(P=0.0002, \mathrm{~N}=24)$, suggesting that searches for foods are strongly correlated with consumption. Thus, we posited that B12 consumption can be estimated through recipe searches.

Awareness of B12 deficiency is also correlated with consumption. The estimated B12 consumption of users was computed by summing the multiplication of the B12 contents per serving of each of the 12 food types by the number of times a user searched about each food type. The average value of the estimated consumption for people who asked about B12 (people who made B12 searches) was $2.407 \mathrm{mcg}$, compared to 2.395 mcg for those who did not, resulting in a $0.5 \%$ difference and a statistically significant ranksum test value $(P<.0001)$. However, the difference in estimated consumption for the 4701 people who specifically asked about B12 deficiency was 2.219 mcg compared to $2.395 \mathrm{mcg}$ for those who did not. This correlated to an $8 \%$ difference and a ranksum test value of $P<.0001$. We interpreted this finding as additional supporting evidence for the claim that estimating B12 consumption through searches for recipes is valid, given the lower estimated B12 consumption for people who indicated an awareness of this deficiency.

The vast majority $(99.94 \%, 8,429,637 / 8,434,338)$ of people in our sample did not ask about B12 deficiency, supplements, or serum level. However, among people who asked about B12 deficiency, 3.83\% (180/4701) asked about B12 supplements (either in the form of dietary supplements or injections), compared to $0.03 \%(2132 / 8,429,637)$ in the population who didn't ask about B12 deficiency. Thus, approximately 151 more times people who asked about deficiency also asked about supplements.

To estimate the correlation between food consumption and the likelihood of asking about each of the medical terms, we constructed an individual-level model where each person is represented through the number of searches they made for recipes containing each of 12 food items and, separately, as to whether they asked about each of the target terms. The behaviors were linked through a linear classification model where the independent terms were the number of searches for each food item and the dependent variables were whether a person asked about the medical term.

The 10 medical terms for which the individual-level model reached the highest goodness of fit $\left(\mathrm{R}^{2}\right)$ value (denoted by $\mathrm{R}^{2} \mathrm{I}$ ), together with the correlation between model coefficients (per food item) and the B12 contents per serving of each food item, denoted by CoB12, are shown in Table 2. The regression coefficients for computing CoB12 are shown in Multimedia Appendix 4. $\mathrm{R}^{2} \mathrm{I}$ values were low even for the best fitting models, but their median value was 78 times greater than $R^{2} I$ values for the control terms (medical terms unassociated with B12 deficiency for the top 10 terms in each list; ranksum $P=0.002$ ). We further noted that $\mathrm{R}^{2} \mathrm{I}$ was correlated with the number of people who asked about each medical condition $(\rho=.86, P<.0001)$. Thus, conditions we hypothesized may be linked to B12 deficiency can indeed be associated with food 
consumption, especially for the more common medical conditions.

Next, we used the models constructed above to estimate the contribution of B12 contents to each medical condition. The correlation between model coefficients and B12 contents of food items (CoB12) was negative for all terms in Table 2, indicating that people searching for B12-rich foods were less likely to search for the medical terms, compared to people searching for B12-poor foods.

Different CoB12 values observed across medical terms can be explained by several factors. First, the average values of the regression coefficients for all but tomatoes were at least 2.3 times greater than those of tomatoes, whose B12 content is negligible. Second, the Spearman correlation between $\mathrm{R}^{2} \mathrm{I}$ and CoB12 was $-.29(P=0.004, \mathrm{~N}=101)$, whereas for the control terms it was non-significant $(\rho=.35, P=0.08, \mathrm{~N}=27)$. Thus, among the target terms, coefficients of better fitting models were larger (more negative) than of poorly fitting models. Finally, CoB12 for medical drug terms was correlated with drug indications, as described below. On average, the correlation for the control terms was only .04, whereas the same number for the target medical terms was, on average -.31 (ranksum $P=.01$ ).
Moreover, positive correlations were only observed for individual control terms and never among the target terms.

The number of possible indications of a drug can affect its correlation with $\mathrm{CoB} 12$, since indications to unrelated conditions may mask the true correlation. We identified the number of indications from the Canadian Adverse Reactions database. Indications were further stratified to identify those indications that contained the term "pain." The number of indications was strongly correlated with the number of people who asked about a medical condition $(\rho=.53, P<.0001, \mathrm{~N}=95)$, but the number of pain indications only marginally significantly correlated with the number of people $(\rho=.29, P=0.048, \mathrm{~N}=95)$. These correlations were likely due to broad-spectrum drugs being offered to treat more conditions than drugs which can only be prescribed to specific indications.

Next, we tested the correlation between the broadness of an indication of a drug on CoB12. We modeled CoB12 of medical drugs as a function of the interaction between the number of people who asked about a term, the number of indications, and the number of pain-related indications using a rank regression model, weighting samples by $\mathrm{R}^{2} \mathrm{I}$. The model reached an $\mathrm{R}^{2}$ of $0.48(P<.0001, \mathrm{~N}=49)$. Statistically significantly correlated coefficients of the model are shown in Textbox 2.

Table 2. $R^{2}$ of the individual-level models to predict the likelihood of asking about medical terms given questions about foods (denoted by $\left.R^{2} I\right)$ and the correlation between model coefficients and B12 contents of these food items for the 10 medical terms with the highest $\mathrm{R}^{2} \mathrm{I}$ values.

\begin{tabular}{llc}
\hline Medical term & $\mathrm{R}^{2} \mathrm{I}^{\mathrm{a}}$ & $\mathrm{CoB}^{\mathrm{b}}$ \\
\hline Gabapentin & 0.003 & -0.405 \\
Tramadol & 0.003 & -0.473 \\
Neuropathy & 0.002 & -0.322 \\
Omeprazole & 0.002 & -0.397 \\
Sertraline & 0.002 & -0.303 \\
Citalopram & 0.002 & -0.333 \\
Oxycodone & 0.002 & -0.269 \\
Duloxetine & 0.002 & -0.329 \\
Trazodone & 0.002 & -0.354 \\
\hline
\end{tabular}

${ }^{\mathrm{a}} \mathrm{R}^{2} \mathrm{I}: \mathrm{R}^{2}$ of the individual-level models to predict the likelihood of asking about medical terms given questions about foods.

${ }^{\mathrm{b}} \mathrm{CoB} 12$ : correlation between model coefficients (per food item) and the B12 contents per serving of each food item.

Textbox 2. Statistically significantly correlated coefficients of the model.

\footnotetext{
The number of indications:

- $\quad$ Slope was $-0.43(P<.0001)$

- Meaning that fewer indications were correlated with higher CoB12

The number of pain indications:

- $\quad$ Slope was $0.20(P=0.04)$

- $\quad$ Meaning that the more pain-related indications, the higher CoB12
}

We interpreted the first as showing that the CoB12 of deficiency was masked by people with other medical conditions. non-specific medicines was lower, as the effect of B12 The second correlation was interpreted as showing that the more 
a drug is used to treat pain (ostensibly caused partly by B12 deficiency), the better the correlation to B12 consumption.

Taken together, our results indicated that the more focused a term is in its medical application, and the more popular, the better the model was in estimating the correlation of B12 consumption with its appearance. The more people consume B12-rich foods, the less likely they were to query for those medical terms we hypothesized were related to B12 deficiency.

\section{Discussion}

\section{Principal Findings}

Our study is the first to employ an analysis of Internet searches to estimate B12 intake and its effect. We showed a strong correlation between food consumption and Internet searches, indicating that searching for recipes reflected food consumption. Our analysis allowed for an assessment of B12 intake from different types of foods, especially meats. The Framingham Offspring study [4] found that B12 intake from meat did not change B12 levels, but this study regarded all types of meat as one entity. Our data, strongly suggested the different types of meat provided different B12 intakes and therefore should be analyzed separately. Most physicians are aware of the risk of B12 deficiency in strict vegans, but the intake from an average non-vegetarian diet is considered sufficient in B12, with no preference to any type of meat. This is likely because an average non-vegetarian diet includes the recommended daily intake. In addition, vitamin B12 stores are high and easy to replenish. Other causes limiting B12 absorption, such as gastric acidity and intrinsic factor deficiency, are considered much more important factors in B12 deficiency. As a result, physicians do not consider non-vegetarians to be at risk for B12 deficiency, with no discrimination between beef- or poultry-based diets. Our study is the first to assess the differences in B12 intake from different sources of meat in daily diet and suggests that the distinction between vegetarians and non-vegetarians may be inadequate for evaluating the risk for B12 deficiency.

Our data also showed that some people are aware of B12 deficiency and that this was reflected in their diet. However, not all patients with B12 deficiency are aware of this shortage. Indeed, difficulties in assessing B12 levels may cause both the patient and clinician to be unaware of its shortage. Here, we showed that low B12 intake was correlated with symptoms associated with B12 deficiency among people who did not search for B12-rich foods, supposedly unaware of B12 deficiency. We performed most of our analyses on a population of people who did not express awareness of B12 deficiency or sufficient intake thereof because patients who are aware of B12 deficiency tend to use supplemental B12, which may mask the effect of dietary B12 intake. Besides further emphasizing the importance of dietary trends, these results suggest that a significant population of patients may suffer from symptomatic B12 deficiency without being aware of the cause of their symptoms. To date, studies evaluating the effect of B12 intake only measured B12 serum levels as an outcome. Our results suggest that other outcomes, such as peripheral neuropathy and other neurological complaints should also be considered.
Our study found a correlation between B12 intake and different drugs and diseases. Paresthesia, which is neuropathic in origin, may be a symptom of B12 deficiency. Not surprisingly, the 4 strongest correlations were related to neuropathy and pain and its treatment. Gabapentin and Lyrica are common treatments for neuropathic pain or central neuropathic pain. Tramadol is also prescribed for neuropathic pain as well as other types of pain. Internet searches for sertraline, citalopram, duloxetine, and trazodone were also inversely correlated with the estimated B12 intake. These drugs are common anti-depressants and our results strengthen the yet controversial association between low B12 levels and depression [22]. Searches for oxycodone, a potent opiate painkiller, were also associated with lower B12 dietary intake. Oxycodone is not the drug of choice for neuropathic pain, but is usually reserved for severe or refractory pain. B12 is not typically associated with non-neuropathic pain, though parenteral B12 has been shown to alleviate low back pain [23]. The association of B12 intake and oxycodone suggested that B12 may have a role in severe or refractory pain which is not neuropathic. Interestingly, omeprazole, an important proton pump inhibitor, was also correlated with decreased B12 dietary intake. The reason for this relationship is unclear. Omeprazole, through its gastric acid lowering effects, is considered to be a cause for B12 deficiency, but B12 deficiency is not known to cause gastric acidity. A possible explanation might be that peptic pain may be amplified in B12 deficiency, but this has never been shown or looked into.

\section{Limitations}

Our study has several limitations. First, a recipe search does not necessarily translate to precise personal consumption, which may have biased the results. We validated our results at the population level (regional consumption), but individual consumption may differ. Past work has found a high correlation between recipe searches and actual consumption [15]. Moreover, in our study we required that recipe searches correlated with the profile of food consumption (ie, the fraction of beef over chicken), not the actual rates of individual consumption. In addition, though correlations were not very high, the fact that despite this limitation we were able to show differences in the use of the target terms as a function of B 12 content (but not in searches unrelated to B12) strengthened the notion that differences in B12 intake from different types of meat are important in determining the risk for B12 deficiency and related symptoms.

The use of Internet searches as a proxy for health conditions may also be biased. For example, B12 has been suggested to be associated with depression, but the depression may cause a decrease in Internet use [24], making it difficult to identify this disorder through Internet queries.

Another limitation is our inability to quantify the impact of dietary B12 on serum levels. Our model identified an increase of risk with consumption of meat with low B12 content, but it cannot predict the serum B12 levels for a specific diet. On the other hand, the difficulties in relying on B12 levels and the occurrence of clinical B12 deficiency alongside seemingly normal serum B12 levels, suggest that assessing symptoms may be more important than estimating serum levels. 
Our work focused on people in the United States. A comparison to other countries and other dietary preferences could enhance our understanding of the effect of B12 intake on pain.

Despite these limitations, utilizing Internet searches as a proxy for B12 intake has the advantage of overcoming memory bias and has the ability to go into dietary details, which is much more difficult to perform in questionnaire-based studies.

\section{Conclusion}

Our data suggest that meat alone is not sufficient to prevent B12 deficiency and that the source of meat should also be considered. Our data also suggests that B12 intake inversely correlates with neurological symptoms, implying a role for B12 among a seemingly unaware population. Physicians should be aware of the possible role of B12 in any patient with neurological complaints or unexplained pain. Further research is necessary to confirm and determine the clinical significance of our results.

\section{Conflicts of Interest}

EYT is an employee of Microsoft, owner of the Bing search engine.

\section{Multimedia Appendix 1}

Terms used in the study.

[PDF File (Adobe PDF File), 331KB-Multimedia Appendix 1]

\section{Multimedia Appendix 2}

Demographics.

[PDF File (Adobe PDF File), 669KB-Multimedia Appendix 2]

\section{Multimedia Appendix 3}

Maps of food consumption.

[PDF File (Adobe PDF File), 342KB-Multimedia Appendix 3]

\section{Multimedia Appendix 4}

Table 2 with regression coefficients for statistically significant interactions.

[PDF File (Adobe PDF File), 482KB-Multimedia Appendix 4]

\section{References}

1. Graham SM, Arvela OM, Wise GA. Long-term neurologic consequences of nutritional vitamin B12 deficiency in infants. J Pediatr 1992 Nov;121(5 Pt 1):710-714. [Medline: 1432418]

2. Lindenbaum J, Healton EB, Savage DG, Brust JC, Garrett TJ, Podell ER, et al. Neuropsychiatric disorders caused by cobalamin deficiency in the absence of anemia or macrocytosis. N Engl J Med 1988 Jun 30;318(26):1720-1728. [doi: 10.1056/NEJM198806303182604] [Medline: 3374544]

3. Almoallim H, Mehdawi FS, Cheikh MM, Al-Dhaheri F, Aqeel AM. Reversible vitamin B12 deficiency presenting with acute cementia, paraparesis, and normal hemoglobin. Case Rep Neurol Med 2016;2016:4301769 [FREE Full text] [doi: 10.1155/2016/4301769] [Medline: 28070432]

4. Tucker KL, Rich S, Rosenberg I, Jacques P, Dallal G, Wilson PW, et al. Plasma vitamin B-12 concentrations relate to intake source in the Framingham Offspring study. Am J Clin Nutr 2000 Feb;71(2):514-522 [FREE Full text] [Medline: 10648266]

5. Healton EB, Savage DG, Brust JC, Garrett TJ, Lindenbaum J. Neurologic aspects of cobalamin deficiency. Medicine (Baltimore) 1991 Jul;70(4):229-245. [Medline: 1648656]

6. Carmel R. How I treat cobalamin (vitamin B12) deficiency. Blood 2008 Sep 15;112(6):2214-2221 [FREE Full text] [doi: 10.1182/blood-2008-03-040253] [Medline: 18606874]

7. Naurath HJ, Joosten E, Riezler R, Stabler SP, Allen RH, Lindenbaum J. Effects of vitamin B12, folate, and vitamin B6 supplements in elderly people with normal serum vitamin concentrations. Lancet 1995 Jul 08;346(8967):85-89. [Medline: $\underline{7603218]}$

8. Komada Y, Narisawa H, Ueda F, Saito H, Sakaguchi H, Mitarai M, et al. Relationship between self-reported dietary nutrient intake and self-reported sleep duration among Japanese adults. Nutrients 2017 Feb 13;9(2):134 [FREE Full text] [doi: 10.3390/nu9020134] [Medline: 28208812] 
9. Gougeon L, Payette H, Morais JA, Gaudreau P, Shatenstein B, Gray-Donald K. Intakes of folate, vitamin B6 and B12 and risk of depression in community-dwelling older adults: the Quebec Longitudinal Study on Nutrition and Aging. Eur J Clin Nutr 2016 Mar;70(3):380-385. [doi: 10.1038/ejen.2015.202] [Medline: 26648330]

10. Almeida OP, Ford AH, Flicker L. Systematic review and meta-analysis of randomized placebo-controlled trials of folate and vitamin B12 for depression. Int Psychogeriatr 2015 May;27(5):727-737. [doi: 10.1017/S1041610215000046] [Medline: 25644193]

11. Vogiatzoglou A, Smith AD, Nurk E, Berstad P, Drevon CA, Ueland PM, et al. Dietary sources of vitamin B-12 and their association with plasma vitamin B-12 concentrations in the general population: the Hordaland Homocysteine Study. Am J Clin Nutr 2009 Apr;89(4):1078-1087 [FREE Full text] [doi: 10.3945/ajen.2008.26598] [Medline: $\underline{19190073}$ ]

12. van Wijngaarden JP, Swart KMA, Enneman AW, Dhonukshe-Rutten RAM, van Dijk SC, Ham AC, et al. Effect of daily vitamin B-12 and folic acid supplementation on fracture incidence in elderly individuals with an elevated plasma homocysteine concentration: B-PROOF, a randomized controlled trial. Am J Clin Nutr 2014 Dec;100(6):1578-1586 [FREE Full text] [doi: 10.3945/ajcn.114.090043] [Medline: 25411293]

13. Yom-Tov E. Crowdsourced Health. Boston: MIT Press; 2016.

14. Brownstein JS, Freifeld CC, Madoff LC. Digital disease detection--harnessing the Web for public health surveillance. N Engl J Med 2009 May 21;360(21):2153-5, 2157 [FREE Full text] [doi: 10.1056/NEJMp0900702] [Medline: 19423867]

15. West R, White R, Horvitz E. From cookies to cooks: insights on dietary patterns via analysis of web usage log. 2013 Presented at: 22nd International Conference on World Wide Web; 2013 May 13-17; Rio de Janeiro, Brazil p. 1399-1410. [doi: $10.1145 / 2488388.2488510]$

16. Trattner C, Parra D, Elsweiler D. Monitoring obesity prevalence in the United States through bookmarking activities in online food portals. PLoS One 2017;12(6):e0179144 [FREE Full text] [doi: 10.1371/journal.pone.0179144] [Medline: 28636665]

17. Abbar S, Mejova Y, Weber I. You tweet what you eat: studying food consumption through twitter. 2015 Presented at: ACM Conference on Human Factors in Computing Systems (CHI 15); 2015 Apr 18-23; Seoul, Republic of Korea p. 3197-3206.

18. Yom-Tov E, Gabrilovich E. Postmarket drug surveillance without trial costs: discovery of adverse drug reactions through large-scale analysis of web search queries. J Med Internet Res 2013 Jun 18;15(6):e124 [FREE Full text] [doi: 10.2196/jmir.2614] [Medline: 23778053]

19. Soldaini L, Yom-Tov E. Inferring individual attributes from search engine queries and auxiliary information. 2017 Presented at: 26th International Conference on World Wide Web; 2017 Apr 3-7; Perth, Australia p. 293-301.

20. Yom-Tov E, Borsa D, Hayward AC, McKendry RA, Cox IJ. Automatic identification of Web-based risk markers for health events. J Med Internet Res 2015 Jan 27;17(1):e29 [FREE Full text] [doi: 10.2196/jmir.4082] [Medline: 25626480]

21. Wikia Recipes. URL: http://recipes.wikia.com/wiki/Recipes Wiki [accessed 2017-12-20] [WebCite Cache ID 6vqCx151s]

22. Coppen A, Bolander-Gouaille C. Treatment of depression: time to consider folic acid and vitamin B12. J Psychopharmacol 2005 Jan;19(1):59-65. [doi: 10.1177/0269881105048899] [Medline: 15671130]

23. Mauro L, Martorana U, Cataldo P, Brancato G, Letizia G. Vitamin B12 in low back pain: a randomised, double-blind, placebo-controlled study. Eur Rev Med Pharmacol Sci 2000;4(3):53-58. [Medline: 11558625]

24. Yom-Tov E, White RW, Horvitz E. Seeking insights about cycling mood disorders via anonymized search logs. J Med Internet Res 2014 Feb 25;16(2):e65 [FREE Full text] [doi: 10.2196/jmir.2664] [Medline: 24568936]

Edited by G Eysenbach; submitted 08.08.17; peer-reviewed by C Trattner, C Basch, X Jiang, KNB Nor Aripin, MN Sadat; comments
to author 19.10.17; revised version received 01.11.17; accepted 04.11.17; published 05.01.18
Please cite as:
Giat E, Yom-Tov E
Evidence From Web-Based Dietary Search Patterns to the Role of B12 Deficiency in Non-Specific Chronic Pain: A Large-Scale
Observational Study
J Med Internet Res $2018 ; 20(1): e 4$
URL: $\underline{\text { http://www.jmir.org/2018/1/e4/ }}$
doi: $\underline{10.2196 / j m i r .8667}$
PMID: $\underline{29305340}$

CEitan Giat, Elad Yom-Tov. Originally published in the Journal of Medical Internet Research (http://www.jmir.org), 05.01.2018. This is an open-access article distributed under the terms of the Creative Commons Attribution License (https://creativecommons.org/licenses/by/4.0/), which permits unrestricted use, distribution, and reproduction in any medium, provided the original work, first published in the Journal of Medical Internet Research, is properly cited. The complete bibliographic information, a link to the original publication on http://www.jmir.org/, as well as this copyright and license information must be included. 